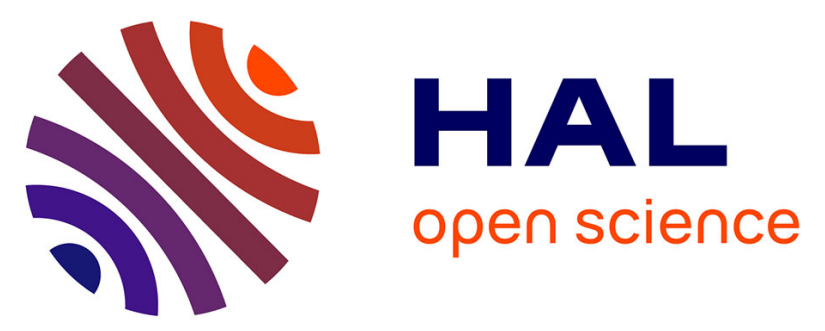

\title{
Automation Experience across Domains: Designing for Intelligibility, Interventions, Interplay and Integrity
}

Peter Fröhlich, Matthias Baldauf, Philippe Palanque, Virpi Roto, Thomas

Meneweger, Manfred Tscheligi, Zoe Becerra, Fabio Paternò

\section{To cite this version:}

Peter Fröhlich, Matthias Baldauf, Philippe Palanque, Virpi Roto, Thomas Meneweger, et al.. Automation Experience across Domains: Designing for Intelligibility, Interventions, Interplay and Integrity. CHI Conference on Human Factors in Computing Systems (CHI 2020), Apr 2020, Honolulu HI, United States. pp.1-8, 10.1145/3334480.3375178 . hal-03099196

\section{HAL Id: hal-03099196 https://hal.science/hal-03099196}

Submitted on 7 Jan 2021

HAL is a multi-disciplinary open access archive for the deposit and dissemination of scientific research documents, whether they are published or not. The documents may come from teaching and research institutions in France or abroad, or from public or private research centers.
L'archive ouverte pluridisciplinaire HAL, est destinée au dépôt et à la diffusion de documents scientifiques de niveau recherche, publiés ou non, émanant des établissements d'enseignement et de recherche français ou étrangers, des laboratoires publics ou privés. 


\section{Automation Experience across Domains: Designing for Intelligibility, Interventions, Interplay and Integrity}

Peter Fröhlich

AIT Austrian Institute of

Technology

Vienna, Austria

peter.froehlich@ait.ac.at

Matthias Baldauf

University of Applied

Sciences St. Gallen

St. Gallen, Switzerland

matthias.baldauf@fhsg.ch

Philippe Palanque

Université Paul Sabatier

Toulouse, France

palanque@irit.fr

\section{Virpi Roto}

Aalto University

Aalto, Finland

virpi.roto@aalto.fi

Thomas Meneweger

University of Salzburg,

Salzburg, Austria

thomas.meneweger@sbg.ac.at

Manfred Tscheligi

University of Salzburg,

Salzburg, Austria \&

AIT Austrian Institute of

Technology, Vienna, Austria

manfred.tscheligi@sbg.ac.at
Zoe M. Becerra

Georgia Institute of Technology

Atlanta, USA

zbecerra3@gatech.edu

Fabio Paternó

CNR-ISTI

Pisa, Italy

fabio.paterno@isti.cnr.it

\begin{abstract}
Automation takes up an increasingly important role in everyday life. The objective of the workshop is to provide a forum for a holistic view on design foundations for automated systems in everyday private, public and professional surroundings. We conceive the workshop as an interdisciplinary forum for usercentered design and research, taking inspiration from diverse problem areas and application fields. Given their current relevance for automation experience, four key aspects (intelligibility, interventions, interplay, and integrity) will be addressed in expert talks, participant presentations and group-wise creative thinking

exercises. The workshop will provide a further step towards a research agenda for comprehensive design and research approaches that provide a transfer and consolidation across different application domains, user requirements and system capabilities.
\end{abstract}

\section{Author Keywords}

Automation; User Experience; Automation Experience;

Human Intervention; Intelligibility; Interplay; Integrity 


\section{Organizers}

\section{Peter Fröhlich}

Senior Scientist at AIT Austrian Institute of

Technology, Center for Technology Experience

Investigates automation experience phenomena in autonomous driving, smart home environments, as well as in industrial production

\section{Matthias Baldauf}

Senior Researcher and Lecturer at the University of Applied Sciences St.Gallen

Expert in Pervasive

Computing and Human-

Computer Interaction with a focus on conversational

assistants for automating processes in various domains

\section{ACM Classification Keywords}

Human-centered computing $\rightarrow$ Human computer interaction $(\mathrm{HCI})$

\section{Background}

Automation has been around us both in the work environment and in the home for a long time. It is by nature pervasive, as it touches each of our critical infrastructures, ranging from water and electricity distribution to escalators and conveyor belts, as well as increasingly information processing and consumption.

However, in comparison to the seamless registration to a WiFi network, triggering the start of the next episode on a video portal or a spam filter removing emails from the inbox, automation is now making its move into everyday life in a different way than before. One of the key characteristics of this novel form of everyday automation is that functions being automated are getting closer to human cognitive functions thus integrating analysis and decision based on the processing of "large" information sets.

Functions being automated are also of higher-leve automation according to the classification from Sheridan and Parasuraman [17], thus blending in one single function many nested functions with possibly conflicting objectives and inconsistent behaviors.

Furthermore, system functions are made programmable and integrateable, requiring more technology skills and knowledge for the end users to be able to benefit from them. Functions are also made accessible from a distance thus requiring from users deep understanding of communication technologies implemented within the various systems. These evolutions shape the everyday automation experience of users and bring multiple issues non-present in previous generation (cf. [15]). For example, more complex automation will build on top of previous knowledge and practice in designing, developing and deploying automation, which will lead to the appearance of unforeseeable failures [20] - with respective consequences on the user experience of such systems.

This workshop aims to provide a forum dedicated to the investigation of ubiquitous automation technologies as they are experienced in everyday situations by nonproficient users. Unlike application-dependent workshops and conferences, we dedicatedly strive to engage with cross-domain, user-centered design challenges and principles of everyday automation experience, thereby considering both foundational work as well as recent scientific discourse (e.g., [8]). Our attempt is to further close the gap of reflection on the experience of automation, and we strive to come up with holistic interaction paradigms for its optimization. The workshop focuses on four key aspects that have been identified in previous research explorations (most importantly the CHI 2019 "Everyday Automation Experience" workshop [6]) as most relevant upcoming user needs: intelligibility, intervention, interplay, and integrity.

\section{Intelligibility}

Emergent automated systems and their underlying data processing are too complex to be fully understood even by experts. This inherent limitation and the increasing number of situations and conditions evolving from the tight embeddedness in everyday home, work and mobility contexts can bring about unintended problems and side effects: unintuitive menus can make cleaning robots start in the middle of the night, the heating 


\section{Organizers (cont.)}

\section{Philippe Palaque}

Professor at University of Toulouse

Expert in safety critical interactions and their engineering

\section{Virpi Roto}

Professor of Practice in Experience Design at Aalto University, Department of Design

Studies the means to design automation that improves user experience even in traditional work contexts. starts when subjectively there is a need for cooling, and machines in the production hall emit a plethora of beep sounds that are indistinguishable by the monitoring staff. Consequently, we need models and design approaches for intelligibility of automated systems, which assist users in understanding their critical states, in forming adequate expectations on their behavior. In this respect, improving intelligibility is the foundation for addressing the challenges of intervention, interplay and integrity discussed below.

Designing for intelligibility should build on and extend the concepts of explainable AI $[1,21]$. An avenue further to explore are displays indicating the awareness and intent of an automated system [7], as well as reliability displays that indicate the degree of uncertainty of a system [10]. Thereby, it is important to consider all possible presentation modalities, including auditory and tactile cues [5]. Corresponding research questions and challenges are as follows:

- How can non-expert users obtain an overall understanding of the reasoning of a system?

- Which styles of communication should be used to convey the state of a ubiquitous automated system?

- How can findings on reliability displays as well as awareness and intent communication be integrated amongst different application domains?

- How to enable people with no or little programming skills to customize the behavior of a system?

\section{Intervention}

With the application of ubiquitous automated systems, the possibility for humans to intervene the systems' processes becomes increasingly crucial. Complex processes, as well as indirect and inexplicit interactions might lead to a loss of control with automated systems, as it is the ultimate goal of automation to reduce explicit user interactions [10]. Explicit input modalities like buttons are replaced with sensors or systems which aim at automatically recognize users' intentions, e.g. During flawless operations of a system this might not be a problem. However, if system errors occur or human interventions are needed, e.g. for system calibration, this might lead to problems with regular user interface paradigms. Given this close relation of automation and user control, Schmidt and Herrmann introduced the paradigm of "intervention user interfaces" [19]. Additionally, interaction paradigms like "peripheral interaction" [3] or "implicit interaction" [11] highlight this shift towards indirect and inexplicit user interaction with ubiquitous automated systems.

Communicating and visualizing the state of the system as well as opportunities for user intervention is

therefore increasingly important. However, designing for human interventions brings along many opportunities as well as challenges for the interaction design of automated systems, such as:

- How to communicate human intervention opportunities and potential consequences?

- How to design for (unobtrusive) awareness of ubiquitous systems' status?

- How to allow human interventions in complex automated procedures?

- To what extend should users be able to overrule the system behavior?

\section{Interplay}

With recent advances in technological fundamentals of automation such as machine learning, interaction with 


\section{Organizers (cont.)}

\section{Thomas Menewege}

PhD student and research fellow at the Center for Human-Computer Interaction, University of Salzburg

Explores workers' experiences and work practices in increasingly automated and interconnected work and production environments (e.g., semiconductor factory, assembly lines, truck driving)

\section{Manfred Tscheligi}

Professor for HCI \& Usability at the University of Salzburg and Head of the Center for Technology Experience at AIT (Vienna)

Leads a variety of research projects investigating automation experience in various contexts (e.g. intelligent production, driving, robotics, and retail) automated systems is no longer restricted to configuring and (potentially) triggering an automated process and consuming its result. Instead the interplay of human users and AI-driven automated systems will be more complex, interweaved, and fine-grained (cf.

[8]). A recent example is Google Duplex [13], which carries out appointment tasks over the phone. The technology can call a restaurant, e.g., and interact with the employee in natural voice. Duplex is an automated system relieving the client of the entire scheduling task and requires the callee to cooperate in a dialogue to reach a joint goal. While Duplex mimics a human caller, a lot of future automated systems in everyday life might be less expressive and thus, making the design of an efficient interplay even more challenging (cf. [2]).

Such an advanced interplay of non-expert users and automated systems raises questions regarding an efficient and intuitive control flow and handover processes. In automotive research, we observe the vision of a tight cooperation and an involvement of the human user in a system's decision-making [22] similarly in automated manufacturing with its "humanin-the-loop" approaches [18] - concepts which seem promising also for other types of everyday automation. The overall task distribution between smart automated systems and humans and the role of human experience and/or creativity are worth investigating [8]. For example, in medicine or social work, autonomous virtual assistants (in form of a mobile app, e.g.) may be used for continuous diagnosis and assistance between appointments with human experts. However, future systems may also provide easily accessible first advise and later pass on to a human expert [4]. Corresponding research questions include:
- How to design for an enjoyable interplay of nonexpert users and automated systems?

- How will future forms of collaboration between nonexpert users and automated systems look like?

- How can non-expert users be involved in decisionmaking processes of ubiquitous automated systems?

- Which are promising applications for collaboration of humans and autonomous systems in everyday life?

\section{Integrity}

By integrity, we refer to trust and ethical aspects of automated systems. Trust in automation is a recurrent topic when user experience or acceptance of

automation is discussed. Emerging research has emphasized the importance of appropriate trust calibration to prevent misuse, disuse, or abuse of an automated system $[9,16]$. Without an appropriate level of trust, people may wrongly rely on or not accept autonomous systems in their everyday life [12]. Trust calibration is not an easy task for users - previous research in professional settings has suggested training on the task may be necessary for interacting with imperfect automation [14]. Therefore, building and maintaining trust is an important design goal of automated systems. Of the manifold factors affecting trust, the features we can design for are related to the system performance and the "design features" [9], of which the latter is relevant in the user interface and interaction design. When designing for trust, the designer can pay attention to appearance, ease-of-use, communication style, transparency, feedback, and the level of control [9]. But are the factors affecting trust in professional contexts the same as in everyday

automation, where training is usually not considered to be important or practical? 


\section{Organizers (cont.)}

\section{Zoe Becerra}

PhD student at the Sonification Lab, Georgia Institute of Technology

Explores factors that impact situation awareness in automated vehicles, such as trust in automation and level of automation

\section{Fabio Paternó}

Research Director at C.N.R.ISTI in Pisa

Investigates user experience aspects of ubiquitous interactive systems, end-user development, adaptive user interfaces
The ethical aspects of automation are often more abstract than the user interface features, consider societal aspects and human rights for example. The ethical guidelines targeted for automation interaction designers are still scarce. A promising avenue to be enforced is to reflect on and formulate guidelines for Human-Automation Interaction by also looking at fairness, ethical concerns and the consideration of diverse cultural backgrounds and norms [2]. Research questions and challenges regarding the integrity of everyday automation include:

- How to design for integrity of automation?

- Is training necessary for interacting with everyday automation?

- What are the interaction design practices to ensure automation integrity in different types of projects and contexts?

- How to assess automation integrity on user interface/interaction level?

\section{Workshop Goals}

This workshop investigates the user experience of automated systems used by non-experts in everyday situations and its design. In line with this overall goal, it will pursue the following subgoals:

- Provide an overview of the diverse field of automation experience and introduce recent research work.

- Reflect on major challenges of designing interactions with ubiquitous (semi-)automated systems and discuss ways to address them.

- Exchange ideas and networking across domains to enable knowledge transfer and best practice exchange regarding the recognition of universa design strategies for ubiquitous automated systems.

- Identify promising future work in the field of everyday automation experience in the form of UX prototypes, project ideas and a research agenda.

\section{Pre-Workshop Plans}

We will announce the workshop through well-known $\mathrm{HCI}$ related mailing lists (CHI Announcements, Ubicomp Announcements, etc.) and suitable websites.

Furthermore, we will send out personal invitations to contact our scientific network directly. All promotional material will include a link to the workshop website (https://everyday-automation.tech-experience.at), which will be set up in time to present the workshop details. We will solicit position papers of up to 5 pages in the ACM SIGCHI Extended Abstract format that describe the participant's workshop contribution. Suitable contribution types include work in progress, concrete research ideas, novel perspectives, and demos that are addressing research questions and challenges of the described four key aspects or complementary pressing issues related to designing interactions with ubiquitous automated systems.

Submissions will be juried by the organizing committee (and additional experts, if needed) on their relevance to the workshop scope, originality, significance and quality. We plan to accept $15-20$ submissions. These papers will be made available on the workshop website before the workshop. Participants will be asked to scan the other participants' position papers as preparation to allow for more productive feedback and in-depth discussions during the workshop. 


\section{Preliminary Schedule}

\section{Morning}

09:00 - 09:15 (15 min.)

Opening \& Welcome

09:15 - 09:40 (25 min.)

Intro: Everyday Automation

[Philippe Palanque]

\section{9:40 - 10:15 (35 min.)}

Session: Intelligibility

[Peter Fröhlich]

10:15 - 10:45 (30 min.)

Coffee Break

10:45 - 11:20 (35 min.)

Session: Intervention

[Thomas Meneweger]

11:20 - 11:55 (35 min.)

Session: Interplay

[Matthias Baldauf]

11:55 - 12:30 (35 min.)

Session: Integrity

[Virpi Roto]

\section{Workshop Structure and Activities}

The workshop is organized as a full-day event. Its program consists of expert talks, participant presentations, creative group works and discussions as well as a joint agenda definition. See the side boxes on pages 6 and 7 for timetables of the preliminary schedule. During the morning session, the organizers and participants will get to know each other in person and share their research perspectives. After a short welcome by the organizing team, an expert talk on automation history and the characteristics of everyday automation will provide a common base for further discussions and inspiration for the creative working session in the afternoon. Following this introduction, the morning session will feature participant presentations of position statements. We plan to have four presentation slots, one for each formerly introduced key aspect of everyday automation, with the participants submissions clustered accordingly.

Each of these four sessions will be introduced by a 5minutes lightning talk of the session chair. Participants will be asked to prepare presentations of their relevant research with a length of about 5 minutes length to create a dynamic and inspiring workshop atmosphere and allow for short Q\&A sessions. Additionally, the audience will be asked to write down challenges, ideas and inspiring aspects that they would like to follow up with in the afternoon. To continue discussions from the morning session, the organizers will propose a nearby restaurant for a joint lunch. Exchanging perspectives and ideas in such an informal setting might foster valuable inspiration for the creative working session in the afternoon. The afternoon session is then dedicated to creative thinking and discussions. Participants will define a topic to drive further in a group creation process, which could either result in design frameworks, prototypes, or project concepts.

To foster results-orientation and efficiency, templates will be provided to the participants for each of these different output types. Participants will also be provided with material for the illustration of their ideas (e.g., sketching papers, post-its, card boards, a camera,

Lego), which they also can take for outdoor discussions on the rooftop patio of the conference venue.

After the coffee break, participants will gather to share their design approaches or project ideas with the overall workshop participant group. The results from the creative thinking phase will then be put into a more coherent form by the workshop organizers and participants. Based on this, an agenda for further research and initiatives will be drawn that shall take into account and build on previous programmatic papers for the whole field of automation, as well as from leading domain-restricted resources and initiatives, such as in automated driving, energy automation and safety-critical systems.

\section{Post-Workshop Plans}

The workshop outcomes and insights will be made available through the workshop website. As a way to take further the agenda discussed in the workshop, opportunities for further initiatives building on the journal special issue on Automation Experience currently prepared in the "Personal and Ubiquitous Computing" journal will be discussed.

\section{Call for Participation}

This one-day workshop provides a multi-disciplinary forum for researchers and practitioners working on 


\section{Preliminary Schedule (cont.)}

\section{Afternoon}

$12: 30$ - 14:00 (90 min.) Lunch Break

14:00 - 14:15 (15 min.)

Intro: Creative Thinking

$14: 15$ - 15:45 (90 min.) Creative Thinking

$\underline{15: 45-16: 15 \text { (30 min.) }}$ Coffee Break

$16: 15$ - 16:45 (30 min.) Result presentations

16:45 - 17:30 (45 min.) Agenda Definition \& Wrap-Up automated systems and their user experience. Participants are asked to submit a position paper describing their recent or future work in the field of designing "everyday automation experiences".

- Position papers must be formatted according to the $\mathrm{CHI}$ Extended Abstract template and comprise up to five pages.

- Position papers must be submitted in PDF format to https://easychair.org/conferences/?conf=automatio nxp20.

- The submissions will be reviewed by the organizers (and additional experts, if required) based on relevance, originality, significance and quality.

- Upon acceptance, at least one author of each accepted position paper must attend the workshop.

- All workshop participants must register for both the workshop and for at least one day of the main conference.

- More details about the workshop can be found at https://everyday-automation.tech-experience.at

\section{Organizers}

The members of the workshop organization team (see left side boxes of pages 2-5) cover a wide spectrum of everyday automation experience research. They have previously organized successful workshops on various

topics of ubiquitous and mobile user experience, as well as automation in transport and safety-critical applications. Furthermore, several members of the committee are experienced workshop and conference chairs. They are known in the HCI and UX communities as long-standing personalities contributing to the overall strategic research agenda, by acting as founding and organizing committee members or chairs for conferences of the abovementioned conferences.

\section{Acknowledgments}

This work is in part supported by the projects "auto.Bus

- Seestadt" (FFG No. 860822) as part of the program

"Mobilität der Zukunft" and MMAssist II (FFG No.

858623) as part of the program "Produktion der

Zukunft" that are operated by the Austrian Research

Promotion Agency FFG. The financial support by the

Austrian Ministry for Transport, Innovation and

Technology is gratefully acknowledged. Furthermore, we would like to acknowledge the support by the project SIM4BLOCKS (funded from the EU's Horizon 2020 research innovation program under grant agreement No. 695965) and the project VA-PEPR (funded by the SNF Sinergia program under grant agreement No. CRSII5_189955).

\section{References}

[1] Ashraf Abdul, Jo Vermeulen, Danding Wang, Brian Y. Lim, and Mohan Kankanhalli. 2018. Trends and Trajectories for Explainable, Accountable and Intelligible Systems: An HCI Research Agenda. In Proc. of CHI'18. ACM.

[2] Saleema Amershi, Dan Weld, Mihaela Vorvoreanu, Adam Fourney, Besmira Nushi, Penny Collisson, Jina Suh, Shamsi Iqbal, Paul N. Bennett, Kori Inkpen, Jaime Teevan, Ruth Kikin-Gil, and Eric Horvitz. 2019. Guidelines for Human-AI Interaction. In Proc. of $\mathrm{CHI}^{\prime} 19 . \mathrm{ACM}$.

[3] Saskia Bakker, Elise Hoven and Berry Eggen. 2015. Peripheral Interaction: Characteristics and Considerations. Personal and Ubiquitous Computing, 19(1), 239-254.

[4] Matthias Baldauf, Stefan Ribler, and Peter Fröhlich. 2019. Alexa, I'm in Need! Investigating the Potential and Barriers of Voice Assistance Services for Social Work. In Proc. of MobileHCI'19. ACM.

[5] Zoe Becerra, Brittany Noah Holthausen and Bruce Walker. 2019. Visual and Auditory Displays of 
Automation Uncertainty. In Proc. of CHI'19 Workshop on Everyday Automation Experience.

[6] Peter Fröhlich, Matthias Baldauf, Thomas Meneweger, Ingrid Erickson, Manfred Tscheligi, Thomas Gable, Boris de Ruyter, and Fabio Paternò. 2019. Everyday Automation Experience: NonExpert Users Encountering Ubiquitous Automated Systems. In EA of $\mathrm{CHI}^{\prime} 19$. ACM.

[7] Peter Fröhlich, Raimund Schatz, Markus Buchta Johann Schrammel, Stefan Suette and Manfred scheligi. 2019. "What's the Robo-Driver up to?" Requirements for Screen-based Awareness and Intent Communication in Autonomous Buses. i-com: Vol. 18, No. 2. Berlin: De Gruyter.

[8] Christian P. Janssen, Stella F. Donker, Duncan P. Brumby, and Andrew L. Kun. 2019. History and Future of Human-Automation Interaction. IJHCS.

[9] Kevin Antony Hoff and Masooda Bashir. 2015 Trust in Automation: Integrating Empirical Evidence on Factors That Influence Trust. Human Factors, 57(3), 407-434.

[10] Lars-Erik Janlert and Erik Stolterman. 2017. Things that Keep Us Busy: The Elements of Interaction. MIT Press, Cambridge, MA, USA.

[11] Wendy Ju and Larry Leifer. 2008. The design of implicit interactions: Making interactive systems less obnoxious. Design Issues, 24(3), 72-84.

12] John D. Lee and Katrina A. See. 2004. Trust in Automation: Designing for Appropriate Reliance. The Journal of the Human Factors and Ergonomics Society 46, 1: 50-80.

[13] Yaniv Leviathan. 2018. Google Duplex: An AI System for Accomplishing Real-World Tasks Over the Phone.

https://ai.googleblog.com/2018/05/duplex-aisystem-for-natural-conversation.html

[14] Stephanie M. Merritt, Deborah Lee, Jennifer L. Unnerstall, and Kelli Huber. 2015. Are well- calibrated users effective users? Associations between calibration of trust and performance on an automation-aided task. Human Factors 57,1:34-47

[15] Philippe Palanque. 2018. Engineering Automations: From a Human Factor Perspective to Design, Implementation and Validation Challenges. In Proc. of EICS'18. ACM.

[16] Raja Parasuraman and Victor Riley. 1997. Humans and Automation: Use, Misuse, Disuse, Abuse. Human Factors 39,2: 230-253.

[17] Thomas B. Sheridan and Raja Parasuraman. 2005. Human-Automation Interaction. Reviews of Human Factors and Ergonomics, vol. 1, no. 1, pp. 89-129.

[18] David Romero, Peter Bernus, Ovidiu Noran, Johan Stahre, Åsa Fast-Berglund. 2016. The Operator 4.0: Human Cyber-Physical Systems \& Adaptive Automation Towards Human-Automation Symbiosis Work Systems. Advances in Production Management Systems. IFIP Advances in Information and Communication Technology, vol 488. Springer, Cham.

[19] Albrecht Schmidt and Thomas Herrmann. 2017. Intervention User Interfaces: A New Interaction Paradigm for Automated Systems. interactions, vol. 24, no. 5, pp. 40-45.

[20] Angelia Sebok and Christopher D. Wickens. 2016. Implementing Lumberjacks and Black Swans Into Model-Based Tools to Support Human-Automation Interaction. Human Factors. 59(2)

[21] Jo Vermeulen. 2014. Designing for Intelligibility and Control in Ubiquitous Computing Environments. Doctoral Thesis, Hasselt University

[22] Marcel Walch, Kristin Mühl, Johannes Kraus, Tanja Stoll, Martin Baumann, and Michael Weber. 2017 From Car-Driver-Handovers to Cooperative Interfaces: Visions for Driver-Vehicle Interaction in Automated Driving. In Automotive User Interfaces. Human-Computer Interaction Series. Springer, Cham 